ApJ LeTTERS, IN PRESS

Preprint typeset using $\mathrm{IATE}_{\mathrm{E}} \mathrm{X}$ style emulateapj v. 04/21/05

\title{
RAM PRESSURE STRIPPING OF AN ISOLATED LOCAL GROUP DWARF GALAXY: EVIDENCE FOR AN INTRA-GROUP MEDIUM
}

\author{
Alan W. McConnachie ${ }^{1}$, Kim A. Venn ${ }^{1}$, Mike J. Irwin ${ }^{2}$, Lisa M. Young ${ }^{3}$, Jonathan J. Geehan ${ }^{1}$ \\ ${ }^{1}$ Department of Physics and Astronomy, University of Victoria, Victoria, B.C., V8P 1A1, Canada \\ ${ }^{2}$ Institute of Astronomy, Madingley Road, Cambridge, CB3 OHA, U.K. and \\ ${ }^{3}$ Physics Department, New Mexico Tech., 801 Leroy Place, Socorro, NM 87801 \\ ApJ Letters, in press
}

\begin{abstract}
We compare the stellar structure of the isolated, Local Group dwarf galaxy Pegasus (DDO 216) with low resolution HI maps from Young et al. (2003). Our comparison reveals that Pegasus displays the characteristic morphology of ram pressure stripping; in particular, the HI has a "cometary" appearance which is not reflected in the regular, elliptical distribution of the stars. This is the first time this phenomenon has been observed in an isolated Local Group galaxy. The density of the medium required to ram pressure strip Pegasus is at least $10^{-5}-10^{-6} \mathrm{~cm}^{-3}$. We conclude that this is strong evidence for an inter-galactic medium associated with the Local Group.

Subject headings: galaxies: dwarf — galaxies: individual (Pegasus, DDO216) — galaxies: interactions — intergalactic medium — Local Group — galaxies: structure
\end{abstract}

\section{INTRODUCTION}

Einasto et al. (1974) first highlighted that dwarf satellites of large galaxies tend to be gas deficient compared to isolated dwarfs. The former generally have little or no ongoing star formation and the stars are pressure supported (dwarf spheroidal, dSph). The latter generally have ongoing star formation and the gas dynamics show that rotational support is important (dwarf irregular, dIrr). "Transition" dwarfs are gas-rich and, unlike dIrr galaxies, have little or no detectable HII regions, although they usually show indications of recent star formation.

The processes by which dwarf galaxies loose their gas are not fully understood. Internal feedback, particularly winds from supernovae, are likely important (Dekel \& Silk 1986) and the existence of the positionmorphology relation clearly indicates that environmental influences are significant. Maver et al. (2006) show that it is possible for dwarf galaxies to be ram pressure stripped of some of their gaseous component in a hot halo of the Milky Way or M31. This idea was originally proposed by Lin \& Faber (1983), who calculated the density of the medium required to be of order $10^{-6} \mathrm{~cm}^{-3}$. There have been no direct detections of such a medium, although recently Nicastro et al. (2002, 2003) and Sembach et al. (2003) have detected OVI absorption which they attribute to hot gas associated with either a Milky Way corona or a Local Group medium.

In this Letter, we compare the stellar and gaseous structure of the isolated, transition-type, dwarf galaxy Pegasus (DDO216). We show that it displays the characteristic signature of ram pressure stripping and conclude that this is strong evidence for hot gas associated with the Local Group. Table 1 summarises some of the observed properties of Pegasus. We adopt the distance estimate by McConnachie et al. (2005),$D \simeq 919 \mathrm{kpc}$, derived from the same photometry used in this Letter.

\section{DATA}

On the night of 8 August 2003, we obtained Johnson $\mathrm{V}\left(V^{\prime}\right)$ and Gunn i $\left(i^{\prime}\right)$ photometry of Pegasus with the
TABLE 1

Summary of observed Parameters for the Pegasus (DDO216) DWARF GALAXY

\begin{tabular}{lll} 
Parameter & Value & Reference \\
\hline$\alpha(\mathrm{J} 2000)$ & $23 \mathrm{~h} 28 \mathrm{~m} \mathrm{36.2 \textrm {s }}$ & - \\
$\delta(\mathrm{J} 2000)$ & $+14^{\circ} 44^{\prime} 35^{\prime \prime}$ & - \\
$(l, b)$ & $\left(94.8^{\circ},-43.6^{\circ}\right)$ & - \\
$M_{V}\left(L_{V}\right)$ & $-12.9\left(1.24 \times 10^{7} L_{\odot}\right)$ & Mateo $(1998)$ \\
$M_{H I}$ & $4.06 \times 10^{6} M_{\odot}$ & Young et al. $(2003)$ \\
$v_{\odot}$ & $-183 \mathrm{~km} \mathrm{~s}^{-1}$ & Young et al. $(2003)$ \\
$v_{r} \sigma$ & 1.7 & Mateo (1998) \\
Distance & $24.82 \pm 0.07(919 \mathrm{kpc})$ & McConnachie et al. (2005) \\
& $24.4 \pm 0.2$ & Gallagher et al. (1998) \\
& $24.9 \pm 0.1$ & Aparicio (1994) \\
\hline
\end{tabular}

Wide Field Camera (WFC) on the 2.5 meter Isaac Newton Telescope (INT), a mosaic of four CCDs with a total field of view of $27 \times 34$ arcmins. This is large enough that the entire dwarf galaxy is contained within a single pointing. The seeing was typically $\lesssim 1.2$ " and exposure times of 1000 seconds in each filter allowed us to reach $i^{\prime} \sim 23.5$ mags and $V^{\prime} \sim 24.5$ with a signalto-noise $\simeq 5$. These data were previously presented in McConnachie et al. (2005), to which we refer the reader for more details. In the central regions of Pegasus, crowding is severe and the photometry is very incomplete; however, this makes no difference to any of the results in this Letter.

The top-left panel of Figure 1 shows the reduced $V^{\prime}$ image of Pegasus taken with the INT WFC. Also shown are the fields of view of previous studies of Pegasus. In particular, the WIYN and HST WFPC2 fields analysed in Gallagher et al. (1998) are shown in green as the largest rectangular field and the small WFPC2 footprint; the NOT field analysed by Aparicio (1994) is shown in blue as the smallest rectangular field, and the field studied by Hoessel \& Mould (1982) using the 1.5m Palomar telescope is shown in red as the medium sized rectangle. As we show below, the extent of Pegasus is significantly larger than has previously been recognised. 
Low resolution HI data for Pegasus was presented in Young et al. (2003) and was obtained using the Very Large Array (VLA) with 2.2 hours in the D array on 13 March 1995 and 12.7 hours in the C array on 24 January 1999. We refer the reader to Young et al. (2003) for details of the data reduction procedures.

\section{ANALYSIS AND DISCUSSION}

\subsection{Comparison of stellar and HI contours}

The top-right panel of Figure 1 shows the tangentplane projection of the spatial distribution of objects identified as stellar from our INT WFC observations of Pegasus. The dotted lines in this panel (and the remaining panels of Figure 1) correspond to the approximate edges of each CCD of the INT WFC. Only objects which lie within $1-\sigma$ of the stellar locus in both the $V^{\prime}$ and $i^{\prime}$-band observations are shown. The hole at the center of the main body of Pegasus is due to severe crowding which causes incompleteness. The bottom-left panel of Figure 1 shows a contour map of the density distribution of stars. The first contour is $2-\sigma$ above the background, and the contours correspond to $2.2,5.0,8.6,13.2,19,0,26.3,35.7,47.5$ and 62.5 stars $\operatorname{arcmin}^{-2}$. The contour map was made in the standard way and follows exactly the methodology described in McConnachie \& Irwin (2006). This panel shows that Pegasus is significantly more extended than suggested by the image in the first panel.

The bottom-right panel of Figure 1 shows the stellar density distribution as a grey-scale with square-root scaling. The red contours are the low-resolution HI distribution from Young et al. (2003). The contours correspond to column densities of $0.1,0.2,0.4,0.8,1.6,3.2,6.4$ and $12.8 \times 10^{20} \mathrm{~cm}^{-2}$. Whereas the stars are distributed in a regular ellipse (typical of a flattened spheroid or an inclined disk) the HI has a "cometary" appearance; the contours in the south-east are more closely packed and do not extend as far as in the north-west.

\subsection{Is Pegasus being ram pressure stripped?}

The shape of the low resolution HI contours in Pegasus - the smooth, compressed contours in the southeast and the "tail" to the north-west - is very similar to the simulated morphology of gas undergoing ram pressure striping (e.g., Stevens et al. 1999; Mori \& Burkert 2000; Marcolini et al. 2003; Roediger \& Hensler 2005; Maver et al. 2006). Observationally, the M81 group dwarf galaxy Holmberg II is observed to have a similar morphology (Bureau \& Carignan 2002), interpreted as evidence of an intra-group medium. In clusters of galaxies, ram pressure stripping of galaxies by an intracluster medium is used to explain various observations, including the deficit of HI in cluster spiral galaxies compared to field spirals (e.g., Giovanelli \& Havnes 1985). Indeed, several individual galaxies in the Virgo Cluster have been shown to display gaseous morphologies indicative of ram-pressure stripping (Vollmer et al. 2000, 2004, 2005).

What else could explain the peculiar appearance of Pegasus? While tidal stripping by large galaxies can affect the structure of dwarf galaxies (e.g., Penarrubia et al. 2007), the closest large galaxy to Pegasus is M31 at $\sim 470 \mathrm{kpc}$ (all the distance estimates in Table 1 place Pe- gasus at $>400 \mathrm{kpc}$ from M31). Even if we assume Pegasus is a weakly-bound satellite of M31, tidal effects at this distance are minimal. If Pegasus was disrupted at pericenter, it is unlikely that the gas would still show signs of current disturbance. Further, tidal stripping tends to produce symmetrical distortions and both gas and stars should be affected. However, these are inconsistent with the structure of Pegasus that we observe.

Could the appearance of Pegasus be due to internal effects rather than external influences? Enhanced star formation in the south-east of Pegasus could produce winds which remove gas from this region. However, if this is the case then the densely packed contours in the south-east should have a more concave, rather than convex, shape. For example, Young et al. (2007) discuss a gas cloud associated with the Phoenix dwarf galaxy and conclude that it was blown out by supernovae winds based in part on the concave shape of its contours.

An alternative explanation for the HI morphology of Pegasus is that it consists of multiple HI clouds, the sum total of which has a cometary appearance. Figure 6 of Young et al. (2003) is a position-velocity diagram of Pegasus along its major axis. It shows a gradient in velocity and two main concentrations of HI which Young et al. (2003) interpret as two distinct HI clouds. The strength of the secondary feature $\left(v \sim-200 \mathrm{~km} \mathrm{~s}^{-1}\right)$ is weaker than the main feature $\left(v \sim-180 \mathrm{kms}^{-1}\right)$ and they join at relatively high column density (between the $8-16-\sigma$ contour levels). An alternative explanation of the data is that the overall velocity gradient is a result of rampressure stripping. The velocity difference between the two features may be due to stripped gas leaving a "hole" in the distribution, making the secondary feature appear at a higher density than its immediate surroundings (we do not necessarily expect that the column density should smoothly vary over the entire cloud).

Henceforth, we adopt the hypothesis that Pegasus is being ram pressure stripped. Following Gunn \& Gott (1972), material will be ram pressure stripped from a galaxy if the density of the surrounding medium, $n_{I G M} \gtrsim$ $\left(2 \pi G \Sigma_{T} \Sigma_{H I}\right) /\left(\mu v^{2}\right) . \quad \Sigma_{T}$ is the total surface density (stars plus gas), $\Sigma_{H I}$ is the column density of $\mathrm{HI}$ and $v$ is the relative velocity of the galaxy to the medium. Thus,

$$
\begin{array}{rl}
n_{I G M} & 3.7 \times 10^{-6} \mathrm{~cm}^{-3}\left(\frac{100 \mathrm{~km} \mathrm{~s}^{-1}}{\mathrm{v}}\right)^{2} \\
& \left(\frac{\Sigma_{H I}}{0.1 \times 10^{20} \mathrm{~cm}^{-2}}\right)^{2},
\end{array}
$$

where we take the mean particle mass $\mu=0.75 m_{p}$ for fully ionized media. We approximate the Local Group space velocity of Pegasus as $v \sim \sqrt{3} \sigma_{L G} \sim 100 \mathrm{kms}^{-1}$ where $\sigma_{L G} \sim 60 \mathrm{~km} \mathrm{~s}^{-1}$ is the Local Group line-of-sight velocity dispersion (Sandage 1986). HI at a column density much lower than $\Sigma_{H I} \sim 0.1 \times 10^{20} \mathrm{~cm}^{-2}$ has been stripped from Pegasus, implying that this is a reasonable lower limit for use in this calculation. We adopt $\Sigma_{T}=$ $\Sigma_{H I}\left(1+M_{\star} / M_{H I}\right)$, where $M_{\star} \sim 1.24 \times 10^{7} M_{\odot}$ is the stellar mass of Pegasus (Table 1). This seems reasonable; the surface brightness of Pegasus is 25 mags $\operatorname{arcsec}^{-2}$ at a radius of $r=1.5^{\prime}$ on the minor axis (Nilson 1973; de Vaucouleurs et al. 1991), corresponding to a stellar surface density of $\Sigma_{\star} \sim 4 \times 10^{20} \mathrm{~cm}^{-2}$. This is approx- 

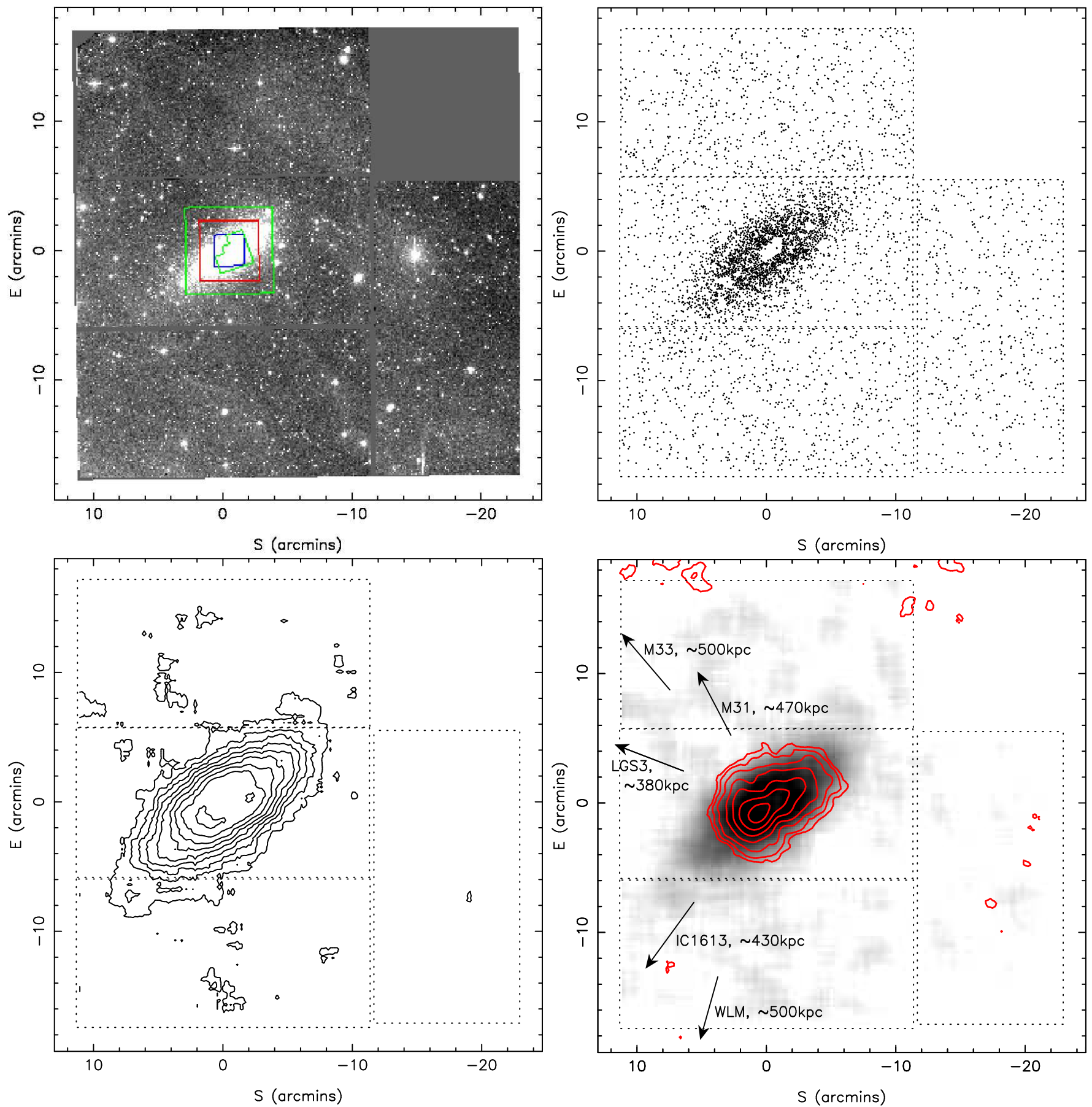

FIG. 1.- Projections in the tangent plane $(\xi, \eta)$ of the structure of the Pegasus dwarf galaxy (DDO216) with the orientation of the field indicated. The dotted lines trace the approximate edges of the four CCDs of the INT WFC. Top-left panel: The reduced $V^{\prime}-$ band image of Pegasus taken with the INT WFC. Also marked are the positions of fields analysed in previous studies of this galaxy; the fields analysed by Gallagher et al. (1998) are shown in green as the largest rectangular field and the small WFPC2 footprint, the field analysed by Aparicio (1994) is shown in blue as the smallest rectangular field, and the field studied by Hoessel \& Mould (1982) is shown in red as the medium sized rectangle. Top-right panel: The distribution of all sources confidently identified as stellar in both the $V^{\prime}$ and $i^{\prime}-$ bands. The hole at the center of Pegasus is due to severe crowding causing the photometry to become seriously incomplete. Bottom-left panel: The stellar density distribution of Pegasus shown as a contour map. The first contour is $2-\sigma$ above the background and the 9 contours correspond to $2.2,5.0,8.6,13.2,19,0,26.3,35.7,47.5$ and 62.5 stars rcmin $^{-2}$. Bottom-right panel: the stellar density distribution is shown as a grey-scale with square-root scaling. The red contours show the low resultion HI distribution from Young et al. (2003). Contours correspond to $0.1,0.2,0.4,0.8,1.6,3.2,6.4$ and $12.8 \times 10^{20} \mathrm{~cm}^{-2}$. Also shown are the projected directions to all galaxies within $\sim 500 \mathrm{kpc}$ of Pegasus which have a significant gaseous content. Pegasus displays the characteristic morphology of ram pressure stripping. 
imately equivalent to the stellar-to-gas mass ratio multiplied by the gas surface density $\left(M_{\star} / M_{H I} \times \Sigma_{H I}\right)$ at $r=1.5^{\prime}$.

These values yield $n_{I G M} \sim 3.7 \times 10^{-6} \mathrm{~cm}^{-3}$. However, given the uncertainties involved, it is entirely plausible that the value of $n_{I G M}$ could be at least an order of magnitude larger than in Equation 1.

\subsection{Consequences}

What is the source of the material that is stripping Pegasus? The bottom right panel of Figure 1 shows the distances of Pegasus to its nearest gas-rich neighbours. The dwarf neighbours are unlikely to be the source of the stripping medium; not only is the required mass of gas unrealistically large (an ejected spherical shell $\sim 1 \mathrm{kpc}$ thick with a radius of $\sim 300 \mathrm{kpc}$ would have a mass $>$ $3 \times 10^{6} M_{\odot}$ at a density of $n_{I G M}$ ) but the energy required is too large for a dwarf galaxy to reasonably provide.

Alternatively, the gas could be associated with M31. From observations of the Magellanic stream, Murali (2000) estimate that the density of the Milky Way halo at the stream must be $\lesssim 10^{-5} \mathrm{~cm}^{-3}$, although Stanimirović et al. (2002) estimate $\sim 10^{-4} \mathrm{~cm}^{-3}$. If the gas density in the halo of M31 is similar, then not only would M31 need to have a very extended corona, but its density would need to decrease very slowly with radius. Indeed, if the Milky Way has a similarly extended corona, then the two will overlap and the result may be observationally indistinguishable from a Local Group medium.

The isolation of Pegasus raises the strong possibility that the stripping medium is associated with the Local Group, rather than individual galaxies within the group. Clusters of galaxies have such media, and observations of Holmberg II imply the presence of an intragroup medium in the M81 group (Bureau \& Carignan 2002). The density of the intra-group medium implied in Equation 1 is of the same order as the density of the medium responsible for local OVI absorption detected by Nicastro et al. (2002, 2003) and Sembach et al. (2003), which they suggest is associated with either a Milky Way corona or a Local Group medium. Our result favors the latter interpretation. Theoretically, $\sim 30 \%$ of baryons in the Local Volume are expected to be in a warm/hot phase ( $T \sim 10^{5}-10^{6} \mathrm{~K}$; Kravtsov et al. 2002); this is likely concentrated around galaxies and galaxy groups as an intra-group medium.

If the stripping medium pervades the Local Group, why do more dwarf galaxies not show evidence of ram pressure stripping? Lin \& Faber (1983) suggest that all the dSphs have been stripped in this fashion, (although Mayer et al. (2006) show that ram-pressure stripping by itself is insufficient to remove all the gas from a dIrr). It is possible that the Local Group medium will be clumpy and perhaps Pegasus is passing through a region of higher density compared to other dIrrs. Alternatively, Pegasus could be falling into and interacting with the Local Group for the first time, as has recently been speculated for two dSph galaxies at large radii from M31 (And XII, Chapman et al. 2007; AndXIV, Majewski et al. 2007). However, the reason why only Pegasus currently shows signs of ram-pressure stripping is unlikely to be known until such time as the masses and orbits of the dIrrs have been determined. Given the distances of these galaxies, this will be some time yet.

\section{SUMMARY}

We show that the isolated, transition-type, Local Group dwarf galaxy, Pegasus (DDO216) is undergoing ram pressure stripping. We calculate that the density of the medium required to strip Pegasus is at least $n_{I G M} \sim$ $10^{-5}-10^{-6} \mathrm{~cm}^{3}$, of the same order as the medium recently identified by Nicastro et al. (2002, 2003) and Sembach et al. (2003) through OVI absorption. Given the large distance of Pegasus from either the Milky Way or M31, we conclude that Pegasus presents strong evidence for the existence of a Local Group inter-galactic medium.

We thank Mary Putman, Stephanie Côté, Evan Skillman, Andi Mahdavi, Arif Babul, and Chris Bildfell for valuable conversations. AWM is supported by a Research Fellowship from the Royal Commission for the Exhibition of 1851, and thanks J. Navarro and S. Ellison for additional financial assistance. KAV and JG thank NSERC for support through a Discovery grant.

\section{REFERENCES}

Aparicio, A. 1994, ApJ, 437, L27

Bureau, M. \& Carignan, C. 2002, AJ, 123, 1316

Chapman, S. C., Peñarrubia, J., Ibata, R., McConnachie, A., Martin, N., Irwin, M., Blain, A., Lewis, G. F., Letarte, B., Lo, K., Ludlow, A., \& O'neil, K. 2007, ApJ, 662, L79

de Vaucouleurs, G., de Vaucouleurs, A., Corwin, Jr., H. G., Buta, R. J., Paturel, G., \& Fouque, P. 1991, Third Reference Catalogue of Bright Galaxies (Volume 1-3, XII, 2069 pp. 7 figs.. SpringerVerlag Berlin Heidelberg New York)

Dekel, A. \& Silk, J. 1986, ApJ, 303, 39

Einasto, J., Saar, E., Kaasik, A., \& Chernin, A. D. 1974, Nature, 252,111

Gallagher, J. S., Tolstoy, E., Dohm-Palmer, R. C., Skillman, E. D., Cole, A. A., Hoessel, J. G., Saha, A., \& Mateo, M. 1998, AJ, 115, 1869

Giovanelli, R. \& Haynes, M. P. 1985, ApJ, 292, 404

Gunn, J. E. \& Gott, J. R. I. 1972, ApJ, 176, 1

Hoessel, J. G. \& Mould, J. R. 1982, ApJ, 254, 38

Kravtsov, A. V., Klypin, A., \& Hoffman, Y. 2002, ApJ, 571, 563

Lin, D. N. C. \& Faber, S. M. 1983, ApJ, 266, L21
Majewski, S. R., Beaton, R. L., Patterson, R. J., Kalirai, J. S., Geha, M. C., Muñoz, R. R., Seigar, M. S., Guhathakurta, P., Bullock, J., Rich, R. M., Gilbert, K. M., \& Reitzel, D. B. 2007, ArXiv Astrophysics e-prints

Marcolini, A., Brighenti, F., \& D'Ercole, A. 2003, MNRAS, 345, 1329

Mateo, M. L. 1998, ARA\&A, 36, 435

Mayer, L., Mastropietro, C., Wadsley, J., Stadel, J., \& Moore, B. 2006, MNRAS, 369, 1021

McConnachie, A. W. \& Irwin, M. J. 2006, MNRAS, 365, 1263

McConnachie, A. W., Irwin, M. J., Ferguson, A. M. N., Ibata, R. A., Lewis, G. F., \& Tanvir, N. 2005, MNRAS, 356, 979

Mori, M. \& Burkert, A. 2000, ApJ, 538, 559

Murali, C. 2000, ApJ, 529, L81

Nicastro, F., Zezas, A., Drake, J., Elvis, M., Fiore, F., Fruscione A., Marengo, M., Mathur, S., \& Bianchi, S. 2002, ApJ, 573, 157

Nicastro, F., Zezas, A., Elvis, M., Mathur, S., Fiore, F., CecchiPestellini, C., Burke, D., Drake, J., \& Casella, P. 2003, Nature, 421,719 
Nilson, P. 1973, Uppsala general catalogue of galaxies (Acta Universitatis Upsaliensis. Nova Acta Regiae Societatis Scientiarum Upsaliensis - Uppsala Astronomiska Observatoriums Annaler, Uppsala: Astronomiska Observatorium, 1973)

Penarrubia, J., Navarro, J. F., \& McConnachie, A. W. 2007, ArXiv e-prints, 708

Roediger, E. \& Hensler, G. 2005, A\&A, 433, 875

Sandage, A. 1986, ApJ, 307, 1

Sembach, K. R., Wakker, B. P., Savage, B. D., Richter, P., Meade, M., Shull, J. M., Jenkins, E. B., Sonneborn, G., \& Moos, H. W. 2003, ApJS, 146, 165

Stanimirović, S., Dickey, J. M., Krčo, M., \& Brooks, A. M. 2002, ApJ, 576, 773
Stevens, I. R., Acreman, D. M., \& Ponman, T. J. 1999, MNRAS, 310,663

Vollmer, B., Balkowski, C., Cayatte, V., van Driel, W., \& Huchtmeier, W. 2004, A\&A, 419, 35

Vollmer, B., Huchtmeier, W., \& van Driel, W. 2005, A\&A, 439, 921 Vollmer, B., Marcelin, M., Amram, P., Balkowski, C., Cayatte, V., \& Garrido, O. 2000, A\&A, 364, 532

Young, L. M., Skillman, E. D., Weisz, D. R., \& Dolphin, A. E. 2007, ApJ, 659, 331

Young, L. M., van Zee, L., Lo, K. Y., Dohm-Palmer, R. C., \& Beierle, M. E. 2003, ApJ, 592, 111 